\title{
Energy source of flagellar type III secretion
}

\author{
Koushik Paul ${ }^{1 *}$, Marc Erhardt $^{1 *} \uparrow$, Takanori Hirano $^{1}$, David F. Blair $^{1} \&$ Kelly T. Hughes ${ }^{1}$
}

\begin{abstract}
Bacterial flagella contain a specialized secretion apparatus that functions to deliver the protein subunits that form the filament and other structures to outside the membrane ${ }^{1}$. This apparatus is related to the injectisome used by many gram-negative pathogens and symbionts to transfer effector proteins into host cells; in both systems this export mechanism is termed 'type III' secretion ${ }^{2,3}$. The flagellar secretion apparatus comprises a membrane-embedded complex of about five proteins, and soluble factors, which include export-dedicated chaperones and an ATPase, FliI, that was thought to provide the energy for export ${ }^{1,4}$. Here we show that flagellar secretion in Salmonella enterica requires the proton motive force (PMF) and does not require ATP hydrolysis by FliI. The export of several flagellar export substrates was prevented by treatment with the protonophore CCCP, with no accompanying decrease in cellular ATP levels. Weak swarming motility and rare flagella were observed in a mutant deleted for FliI and for the nonflagellar type-III secretion ATPases InvJ and SsaN. These findings show that the flagellar secretion apparatus functions as a protondriven protein exporter and that ATP hydrolysis is not essential for type III secretion.
\end{abstract}

Flagellar assembly begins with structures in the cytoplasmic membrane and proceeds through steps that add the exterior structures in a proximal-to-distal sequence (Fig. 1) ${ }^{1}$. Assembly of the rod, hook and filament requires the action of the secretion apparatus, which transports the needed subunits into a central channel through the structure that conducts them to their site of incorporation at the tip (Fig. 1). Flagellar export is notably fast: in the early stages of filament growth flagellin is delivered at a rate of several $55 \mathrm{kDa}$ subunits per second ${ }^{5}$.

ATP hydrolysis by FliI was thought to provide the energy for export because mutations that delete or reduce the activity of FliI block flagellar synthesis at the stage of rod assembly ${ }^{1,4,6}$ (Fig. 1). Homologues of FliI also occur in the type III secretion apparatus of injectisomes and are usually assumed to energize export in those systems as well. Some evidence for a different view has also been reported: it was observed that type III secretion in Yersinia enterocolitica was prevented by the protonophore $\mathrm{CCCP}^{7}$, and it was shown that the secretion ATPase InvC of Salmonella functions to dissociate export substrate from the chaperone ${ }^{8}$, a role distinct from transport itself. The energy source for type III secretion thus remains uncertain.

To address the energy requirements for type III secretion, we first measured the effect of the uncoupler CCCP on flagellar export in $S$. enterica, assayed by accumulation of the export substrate FlgM in the medium. FlgM export was prevented by $10 \mu \mathrm{M}$ or more CCCP (Fig. 2a). Overall cellular energy levels seemed unaffected, because cells grew normally in $10 \mu \mathrm{M}$ CCCP (growth data not shown) and ATP levels were unchanged (Supplementary Fig. 1). The effect was reversible: FlgM export was largely restored following a 30-min washout of the $10 \mu \mathrm{M}$ CCCP (Fig. 2b). FlgM inhibits transcription of its own gene, and so reduced FlgM export might partially reflect decreased cellular levels of the protein ${ }^{9}$. To circumvent this autoinhibitory effect the experiment was repeated with a strain in which flgM was placed under control of a non-native $\left(\mathrm{P}_{\text {arab }}\right)$ promoter. In this strain, the cytoplasmic level of FlgM remained nearly constant, whereas FlgM secretion was again prevented by $10 \mu \mathrm{M}$ or more CCCP (Fig. 2c).

The maintenance of normal ATP levels in the presence of CCCP was noted previously in experiments with $Y$. enterocolitica ${ }^{7}$ and is thought to be due to a regulatory mechanism that inhibits the hydrolytic activity of the ATP synthase when the membrane is deenergized $^{10}$. Because this protective mechanism may not act instantaneously, cellular ATP levels might undergo a transient drop following CCCP treatment that would escape detection in our measurements. To rule out such an effect, we measured FlgM export in a $\triangle a t p A$ strain that lacks a major subunit of the ATP synthase. FlgM secretion was again prevented by $10 \mu \mathrm{M}$ or higher CCCP, and ATP levels were unaffected (Fig. 2d, e).

The flagellum exports more than a dozen substrates, which are classified as early or late according to whether they are secreted during assembly of the hook/basal-body or the filament ${ }^{1}$ (Fig. 1). To test the generality of the PMF requirement we examined the effect of CCCP on secretion of additional substrates, assayed by their accumulation in the culture medium ${ }^{11}$. We observed the early substrate FliK and the late-export substrates FlgK, FlgL and FliC in culture supernatants of LB-grown cells. Accumulation of all four substrates was prevented by treatment with $20 \mu \mathrm{M}$ CCCP (Fig. 2f). The band between FliC and FlgK does not correspond to the size of a known flagellar protein and is likely to be a stable protein secreted in (relatively) low amounts.

CCCP functions as a proton carrier to discharge both the electric potential $(\Delta \psi)$ and concentration $(\Delta \mathrm{pH})$ components of the PMF. To examine the contribution of the $\Delta \psi$ component separately, we measured FlgM secretion in cells treated with the $\mathrm{K}^{+}$-ionophore valinomycin. In medium containing $150 \mathrm{mM} \mathrm{KCl}$, FlgM secretion was inhibited by $10 \mu \mathrm{M}$ valinomycin (Fig. 3a). Thus, the electrical potential component seems essential for export under the conditions of this experiment (extracellular $\mathrm{pH}=7.3$ ). Next, the $\Delta \mathrm{pH}$ component of the gradient was discharged by the weak acid acetate $(34 \mathrm{mM})$, which crosses the membrane in neutral (protonated) form and releases protons inside the cell. At an external $\mathrm{pH}$ of 7, FlgM secretion was not affected by treatment with acetate (Fig. 3b), indicating that flagellar export can be supported by $\Delta \psi$ alone. At an external $\mathrm{pH}$ of 5 , acetate prevented secretion (Fig. 3b), presumably owing to acidification of the cytoplasm and the resulting protonation of one or more functionally important acidic groups. A similar effect was reported with the flagellar motor, which ceased rotating when the cytosolic $\mathrm{pH}$ was lowered to 5 (ref. 12).

We characterized export requirements further using an assay based on secretion of a FlgE-Bla (hook/ $\beta$-lactamase) fusion protein. Cells were deleted for the rod proteins FlgB and FlgC (Fig. 1) to direct the 
fusion protein into the periplasm, allowing export to be quantified by the MIC (minimum inhibitory concentration) for ampicillin ${ }^{13}$. The MIC value was reduced by uncoupler, from a value of $25 \mu \mathrm{g} \mathrm{ml}^{-1}$ in the untreated control to about $4 \mu \mathrm{g} \mathrm{ml}^{-1}$ in $30 \mu \mathrm{M}$ CCCP (Table 1, and Supplementary Information).

If energy for flagellar transport comes from the proton gradient, then ATP hydrolysis by FliI may be less important than has been supposed. To examine the FliI requirement more closely we

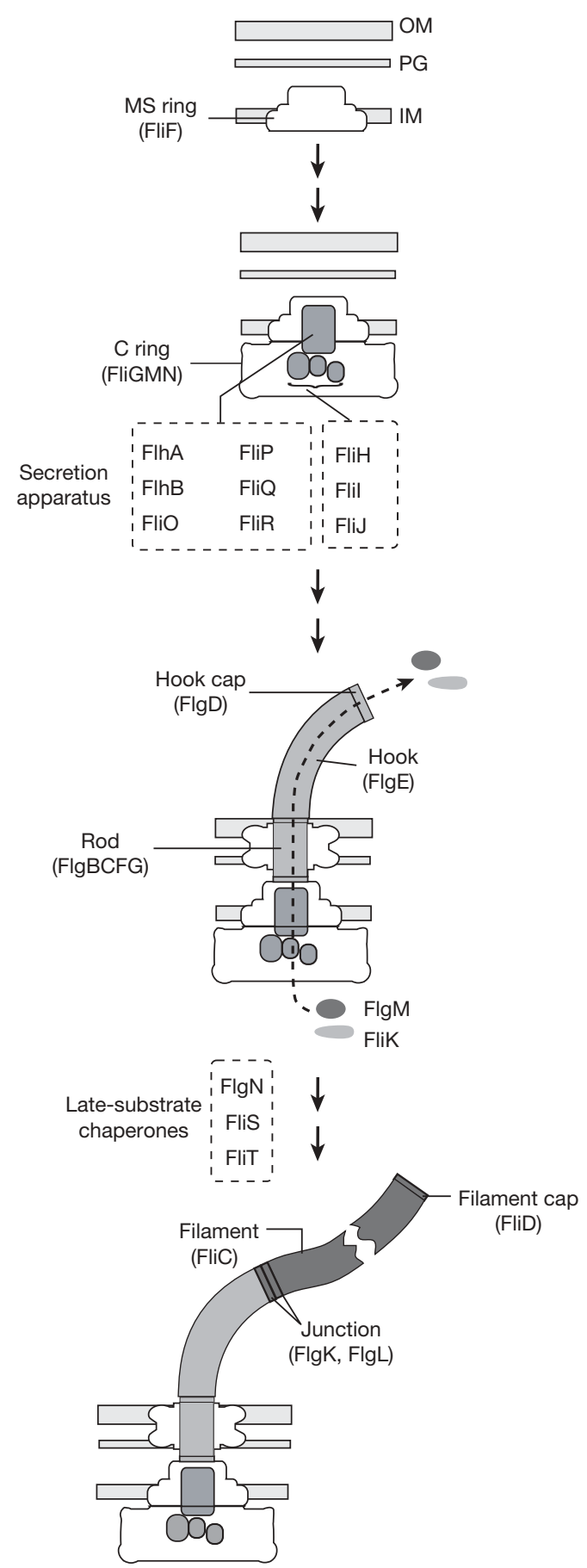

Figure 1 Steps in flagellar assembly. Dashed boxes indicate the proteins that function in flagellar secretion, either in the membrane-bound part of the apparatus or in delivery of substrate. Flagellar components that depend on export are indicated in light- (early substrates) or dark- (late substrates) grey; these include the structural proteins that form the rod, hook and filament, the transcriptional regulator FlgM, and the hook-length regulator FliK. OM, outer membrane; PG, peptidoglycan; CM, cytoplasmic membrane. measured FlgE-Bla export in a strain deleted for fliI and the flanking genes fliH and fliJ. FliH is a regulator of FliI ${ }^{14}$ and FliJ interacts with the FliHI complex and with other export components ${ }^{15,16}$. The MIC measured for the $\Delta$ fliHIJ strain was $12 \mu \mathrm{g} \mathrm{ml}^{-1}$, reproducibly larger than that of a negative-control strain lacking the MS-ring gene fliF $\left(<3 \mu \mathrm{g} \mathrm{ml}^{-1}\right)$ or a strain with all the flagellar genes repressed by
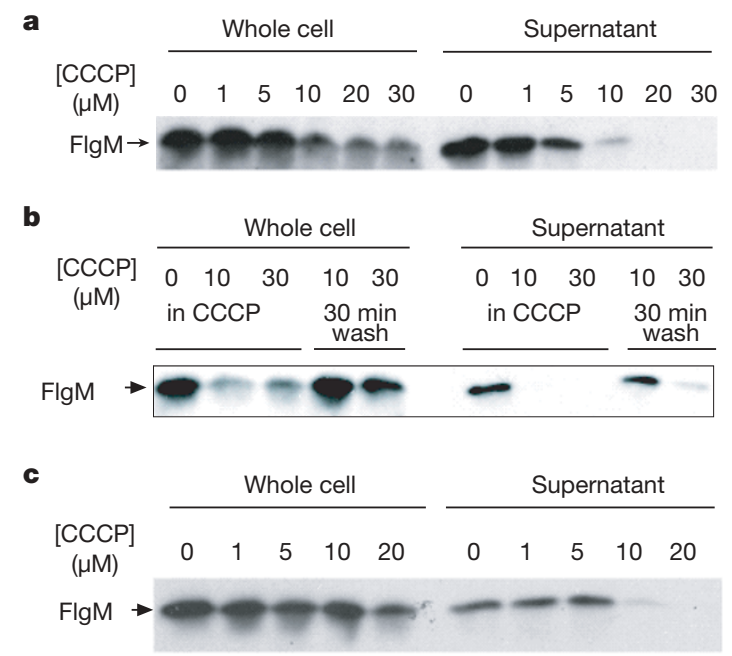

d
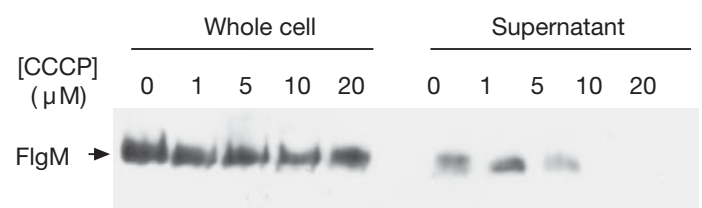

e

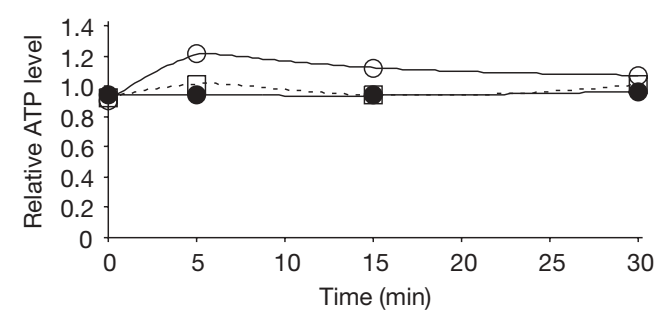

f

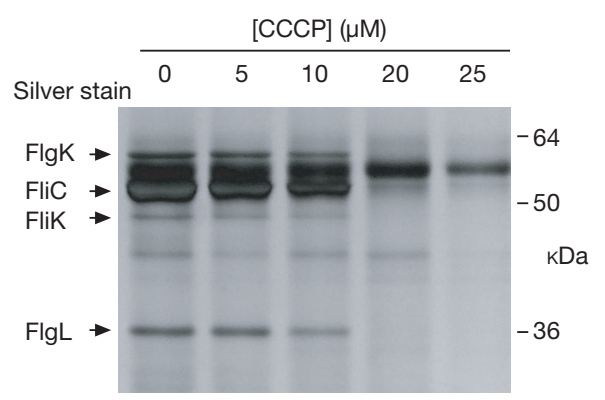

FliK immunoblot

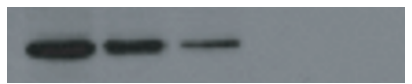

Figure 2 | Inhibition of FlgM secretion by CCCP. a, Secretion in Salmonella strain TH3730 (Tet-inducible flhDC). b, Partial restoration of export following a 30-min wash into CCCP-free buffer. c, Secretion in strain TH10874 (arabinose-inducible flgM). d, Inhibition of FlgM secretion by CCCP in an ATP-synthase defective ( $\triangle a t p A)$ strain (TH11802). e, ATP levels in the $\triangle a t p A$ mutant at various times following treatment with CCCP. Open circles, no treatment; open square, $10 \mu \mathrm{M}$ CCCP; and filled circles, $30 \mu \mathrm{M}$ CCCP. f, Inhibition of secretion of other flagellar substrates (FlgK, FlgL, FliC and FliK) by CCCP. 
a

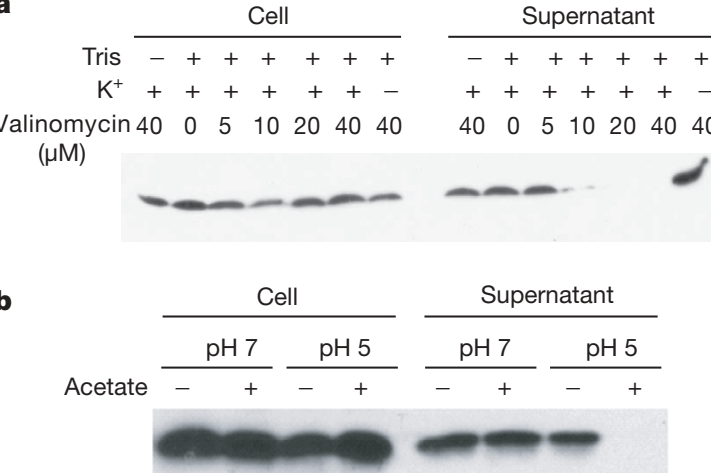

Figure 3 | Effect of $\Delta \psi$ and $\Delta \mathrm{pH}$ on FlgM export. a, Inhibition of FlgM secretion by valinomycin and $\mathrm{K}^{+}$. Where indicated, cells were pretreated with Tris $(120 \mathrm{mM})$ to permeabilize the outer membrane to valinomycin. b, Inhibition of FlgM secretion by acetate $(34 \mathrm{mM})$ at $\mathrm{pH} 5$.

downregulation of the master regulators $\operatorname{flhDC}\left(<3 \mu \mathrm{g} \mathrm{ml}^{-1}\right)$ (Table 1). Furthermore, the MIC value of the $\Delta$ fliHIJ strain was greatly increased (to $800 \mu \mathrm{g} \mathrm{ml}^{-1}$ ) by overexpression of FliR, a membrane-associated part of the export apparatus (Table 1, and Supplementary Data). Prompted by this evidence of export in the absence of FliI, we examined motility of $\Delta$ fliHIJ cells. The $\Delta$ fliHIJ cells migrated in soft agar at about one-tenth the wild-type rate (Fig. 4a), and a fraction of the cells were observed to swim in liquid media. Cells isolated from the centre, edges or intermediate positions in the swarm showed the same phenotype when purified and re-tested (not shown), and so the slow motility is a property of the population and is not due to suppressing mutations. Staining showed flagella on a small fraction $(<1 \%)$ of the cells (Fig. 4 b, left panel). A $\Delta$ fliI mutant swarmed more slowly than the $\Delta$ fliHIJ strain (Fig. 4a) and also showed reduced export in the MIC assay (Table 1), consistent with the more severe motility defect reported previously for a $\Delta$ fli mutant $t^{4,17}$.

In addition to the flagella apparatus, members of Salmonella spp. contain two non-flagellar (injectisome) type III secretion systems, with associated ATPases InvC and $\mathrm{SsaN}^{8,18}$. To rule out any involvement of InvC or SsaN in the secretion observed in $\Delta$ fliHIJ cells, we repeated the experiments in a $\Delta i n v C \Delta s s a N \Delta f l i H I J$ strain. The tripledeletion mutant swarmed equally as well as the $\Delta$ fliHIJ strain in soft agar (Fig. 4a), and flagella were again seen on a few cells (Fig. 4b, right). Thus, none of the secretion ATPases is required for flagellar export, assembly or function.

Our conclusions are consistent with previous observations of a PMF requirement for flagellar growth from more than 25 years ago $^{19}$, and extend the earlier findings in showing that export can be energized by PMF alone in the absence of any type III secretion ATPase $^{7}$. Use of the proton gradient is perhaps not surprising given the speed of type III secretion and the likely advantage of tapping a proximal energy source. Rapid subunit export presumably requires a

\section{Table 1 | Effects of CCCP and mutation on flagellar export}

\begin{tabular}{|c|c|}
\hline Genotype (treatment) & $\mathrm{MIC}\left(\mu \mathrm{g} \mathrm{ml}^{-1}\right)^{*}$ \\
\hline$\Delta f \lg B C$ flgE:bla & 25 \\
\hline$\Delta$ flgBC flgE:bla $(30 \mu \mathrm{M}$ CCCP) & $<3$ \\
\hline$\triangle$ flgBC flgE:bla Tet-inducible flhDC (no Tet) & $<3$ \\
\hline$\Delta$ flgBC flgE:bla Tet-inducible flhDC (15 $\left.\mu \mathrm{g} \mathrm{ml}^{-1} \mathrm{Tet}\right)$ & 50 \\
\hline$\Delta$ flgBC flgE:bla Tet-inducible flhDC, $\Delta$ fliF $\left(15 \mu \mathrm{g} \mathrm{ml} \mathrm{m}^{-1}\right.$ Tet $)$ & $<3$ \\
\hline$\Delta$ flgBC flgE:bla $\Delta$ fliHIJ & 12 \\
\hline$\Delta$ flgBC flgE:bla $\Delta$ fliHIJ, fliR expressed from plasmid & 800 \\
\hline$\Delta$ flgBC flgE:bla $\Delta$ flil & 6 \\
\hline$\Delta$ flgBC flgE:bla $\Delta$ fliP & 4.4 \\
\hline$\Delta$ flgBC flgE:bla $\Delta$ fliP, fliR on plasmid & 4.4 \\
\hline$\Delta$ flgBC flgE:bla $\Delta$ fliP, fliP on plasmid & 800 \\
\hline
\end{tabular}

* MIC, minimum inhibitory concentration of ampicillin required in cells with rod-gene ( $f(g B C)$ deletions to direct the hook- $\beta$-lactamase (FlgE-Bla) fusion protein into the periplasm ${ }^{13}$ (further details are provided in Supplementary Information). a
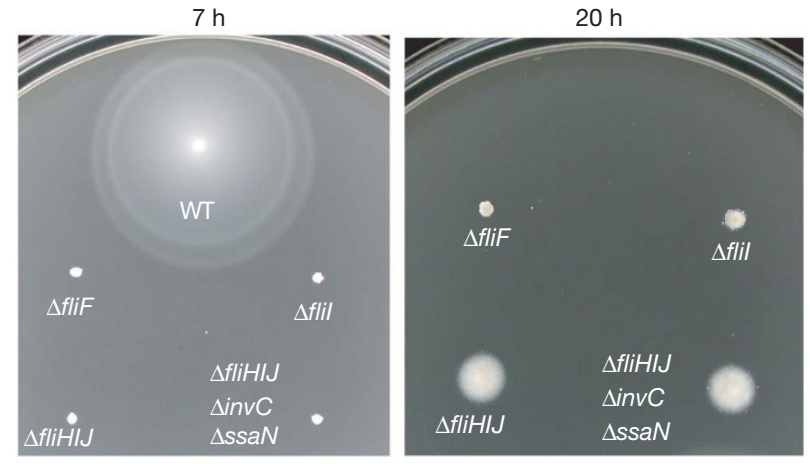

b
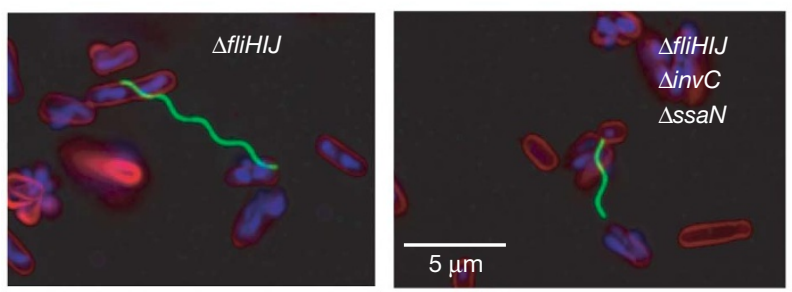

Figure 4 | Flil is non-essential for flagellar assembly and function.

a, Swarming of the $\Delta$ fliHIJ deletion strain and a $\Delta$ fliHIJ $\Delta i n v C \Delta s s a N$ strain with all type-III secretion ATPases deleted. The $\Delta$ fliF strain, blocked in the earliest step of flagellar assembly (Fig. 1), is included as a negative control. Plates were incubated at $32^{\circ} \mathrm{C}$. b, Flagella on cells of the $\Delta$ fliHIJ mutant (left panel) and the $\Delta$ fliHIJ $\Delta i n v C \Delta s s a N$ triple-deletion (right panel). Flagella were visualized with FITC-conjugated anti-FliC antibody (green) ${ }^{26}$. DNA was stained with DAPI (blue), and membranes by FM64 (red).

rapid supply of energy, which might be more easily delivered by a proton current than by ATP hydrolysis. Given that type III secretion is energized by PMF, future studies should focus on the molecular mechanism of proton movement through the apparatus and its coupling to movement of substrate.

\section{METHODS SUMMARY}

Media, growth conditions, methods for phage-mediated transduction and motility assays were performed as described previously ${ }^{9,20,21}$. Carbonylcyanide $m$-chlorophenylhydrozone (CCCP) and valinomycin were from Sigma (analytical grade). Potassium acetate was from J. T. Baker, growth media from Difco, and buffers from Sigma.

FlgM secretion was assayed by accumulation of the protein in the culture medium, using procedures described previously ${ }^{22}$ with minor modifications described in Methods. Cells at mid-log growth stage were treated with the PMF-discharging agents (CCCP or valinomycin) at the concentrations indicated in the figures, introduced from freshly prepared stocks. Following centrifugation to remove cells, supernatants were filtered and FlgM was detected by immunoblotting, essentially as described ${ }^{23}$.

ATP was measured using the firefly luciferase assay and sample-processing procedures of ref. 24.

Ampicillin resistance in FlgE-Bla-exporting cells was assayed as described ${ }^{13}$, with minor modifications described in Methods. Briefly, cells were diluted to $D_{600}=0.01$, cultured for $4.5 \mathrm{~h}$ in LB media containing ampicillin in a series of dilutions ranging from $800 \mu \mathrm{g} \mathrm{ml}^{-1}$ to $1.56 \mu \mathrm{g} \mathrm{ml}^{-1}$, and the minimum inhibitory concentration (MIC) was taken as the lowest ampicillin concentration giving $D_{600}<0.05$. The periplasmic fractionation and anti-Bla immunoblot detection of FlgE/Bla in the cellular and periplasmic fractions was performed as described by ref. 25 .

Swarming motility was assayed in plates containing tryptone broth and $0.28 \%$ bacto-agar. Plates were incubated at $32{ }^{\circ} \mathrm{C}$. Flagellar immunostaining used the methods of ref. 26

Full Methods and any associated references are available in the online version of the paper at www.nature.com/nature.

Received 7 August; accepted 23 November 2007.

1. Macnab, R. M. How bacteria assemble flagella. Annu. Rev. Microbiol. 57, 77-100 (2003). 
2. Cornelis, G. R. The type III secretion injectisome. Nature Rev. Microbiol. 4, 811-825 (2006).

3. Blocker, A., Komoriya, K. \& Aizawa, S. Type III secretion systems and bacterial flagella: insights into their function from structural similarities. Proc. Natl Acad. Sci. USA 100, 3027-3030 (2003)

4. Fan, F. \& Macnab, R. M. Enzymatic characterization of Flil. An ATPase involved in flagellar assembly in Salmonella typhimurium. J. Biol. Chem. 271, 31981-31988 (1996).

5. lino, T. Assembly of Salmonella flagellin in vitro and in vivo. J. Supramol. Struct. 2, 372-384 (1974).

6. Vogler, A. P., Homma, M., Irikura, V. M. \& Macnab, R. M. Salmonella typhimurium mutants defective in flagellar filament regrowth and sequence similarity of Flil to $\mathrm{F}_{0} \mathrm{~F}_{1}$, vacuolar, and archaebacterial ATPase subunits. J. Bacteriol. 173, 3564-3572 (1991).

7. Wilharm, G. et al. Yersinia enterocolitica type III secretion depends on the proton motive force but not on the flagellar motor components MotA and MotB. Infect. Immun. 72, 4004-4009 (2004).

8. Akeda, Y.\& Galan, J. E. Chaperone release and unfolding of substrates in type III secretion. Nature 437, 911-915 (2005).

9. Gillen, K. L. \& Hughes, K. T. Negative regulatory loci coupling flagellin synthesis to flagellar assembly in Salmonella typhimurium. J. Bacteriol. 173, 2301-2310 (1991).

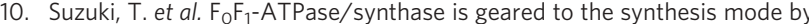
conformational rearrangement of $\varepsilon$ subunit in response to proton motive force and ADP/ATP balance. J. Biol. Chem. 278, 46840-46846 (2003).

11. Komoriya, K. et al. Flagellar proteins and type III-exported virulence factors are the predominant proteins secreted into the culture media of Salmonella typhimurium. Mol. Microbiol. 34, 767-779 (1999).

12. Minamino, T., Imae, Y., Oosawa, F., Kobayashi, Y. \& Oosawa, K. Effect of intracellular $\mathrm{pH}$ on rotational speed of bacterial flagellar motors. J. Bacteriol. 185, 1190-1194 (2003)

13. Lee, H. J. \& Hughes, K. T. Posttranscriptional control of the Salmonella enterica flagellar hook protein FlgE. J. Bacteriol. 188, 3308-3316 (2006).

14. Minamino, T. \& MacNab, R. M. FliH, a soluble component of the type III flagellar export apparatus of Salmonella, forms a complex with Flil and inhibits its ATPase activity. Mol. Microbiol. 37, 1494-1503 (2000).

15. Fraser, G. M., Gonzalez-Pedrajo, B., Tame, J. R. \& Macnab, R. M. Interactions of FliJ with the Salmonella type III flagellar export apparatus. J. Bacteriol. 185, 5546-5554 (2003).

16. Evans, L. D., Stafford, G. P., Ahmed, S., Fraser, G. M. \& Hughes, C. An escort mechanism for cycling of export chaperones during flagellum assembly. Proc. Natl Acad. Sci. USA 103, 17474-17479 (2006).

17. Dreyfus, G., Williams, A. W., Kawagishi, I. \& Macnab, R. M. Genetic and biochemical analysis of Salmonella typhimurium Flil, a flagellar protein related to the catalytic subunit of the $\mathrm{F}_{0} \mathrm{~F}_{1}$ ATPase and to virulence proteins of mammalian and plant pathogens. J. Bacteriol. 175, 3131-3138 (1993).

18. Hensel, M. et al. Functional analysis of ssaJ and the ssaK/U operon, 13 genes encoding components of the type III secretion apparatus of Salmonella pathogenicity island 2. Mol. Microbiol. 24, 155-167 (1997).

19. Galperin, M., Dibrov, P. A. \& Glagolev, A. N. $\Delta \mu \mathrm{H}^{+}$is required for flagellar growth in Escherichia coli. FEBS Lett. 143, 319-322 (1982).

20. Davis, R. W., Botstein, D. \& Roth, J. R. Advanced Bacterial Genetics (Cold Spring Harbor Laboratory, Cold Spring Harbor, NY, 1980).

21. Gillen, K. L. \& Hughes, K. T. Molecular characterization of flgM, a gene encoding a negative regulator of flagellin synthesis in Salmonella typhimurium. J. Bacteriol. 173, 6453-6459 (1991).

22. Karlinsey, J. E., Tsui, H.-C. T., Winkler, M. E. \& Hughes, K. T. Flk couples flgM translation to flagellar ring assembly in Salmonella typhimurium. J. Bacteriol. 180, 5384-5397 (1998).

23. Hughes, K. T., Gillen, K. L., Semon, M. J. \& Karlinsey, J. E. Sensing structural intermediates in bacterial flagellar assembly by export of a negative regulator. Science 262, 1277-1280 (1993).

24. Bakker, E. P. \& Mangerich, W. E. The effects of weak acids on potassium uptake by Escherichia coli K-12 inhibition by low cytoplasmic pH. Biochim. Biophys. Acta 730, 379-386 (1983)

25. Aldridge, P., Karlinsey, J. E., Becker, E., Chevance, F. F. \& Hughes, K. T. Flk prevents premature secretion of the anti-sigma factor FlgM into the periplasm. Mol. Microbiol. 60, 630-643 (2006).

26. Rosu, V. \& Hughes, K. T. $\sigma^{28}$-dependent transcription in Salmonella enterica is independent of flagellar shearing. J. Bacteriol. 188, 5196-5203 (2006).

27. Laemmli, U. K. \& Favre, M. Maturation of the head of bacteriophage T4. I. DNA packaging events. J. Mol. Biol. 80, 575-599 (1973).

Supplementary Information is linked to the online version of the paper at www.nature.com/nature.

Acknowledgements We thank S. Williams for his help and permission for using the luminometer, F. Chevance for advice and assistance with strain constructions, M. Sarkar for assistance with MIC assays, and V. Clougherty for flagellar-staining experiments. M. Erhardt gratefully acknowledges scholarship support of the Studienstiftung des deutschen Volkes. This work was supported by Public Service grants (K.T.H. and D.F.B.) from the National Institutes of Health.

Author Information Reprints and permissions information is available at www.nature.com/reprints. Correspondence and requests for materials should be addressed to D.F.B. (blair@bioscience.utah.edu) and K.T.H. (hughes@biology.utah.edu). 


\section{METHODS}

FlgM-secretion assay. FlgM secreted into the culture medium was measured as described previously ${ }^{2}$, with the following modifications. Strain TH3730 (Tcinduced flagellar synthesis) was cultured overnight at $37^{\circ} \mathrm{C}$ in $\mathrm{LB}$ media, then diluted 100 -fold into $3 \mathrm{ml}$ of fresh LB and cultured to $a n D_{600}=0.5$. Tetraycline $\left(15 \mu \mathrm{g} \mathrm{ml}^{-1}\right)$ was added to induce transcription of the flagellar regulatory genes flhDC and growth was continued for one hour at $37^{\circ} \mathrm{C}$ before cells were harvested. Strain LT2 was cultured in the same way except without tetracycline induction and harvested at an $D_{600}=0.8$. Cells were collected by centrifugation and washed twice with $3 \mathrm{ml} \mathrm{LB}$ containing appropriate concentrations of the PMF-discharging agents (CCCP or valinomycin), introduced from freshly prepared stocks in DMSO. Controls received only DMSO. Following resuspension the cells were incubated at $37^{\circ} \mathrm{C}$ for the times indicated in the figures (typically $30 \mathrm{~min})$, then kept on ice until further processing. Strains TH10874 and TH11802 were treated in a similar way, except media included $0.2 \%$ $\mathrm{L}$-arabinose to induce transcription of $f \mathrm{lg} M$.

To quantify FlgM in the culture medium, cells of a 2-ml sample were pelleted in a tabletop centrifuge and $1.8 \mathrm{ml}$ of the supernatant was passed through a $0.45-\mu \mathrm{m}$ cellulose acetate filter (Nalgene) to remove any remaining cells. Filtrate $(1.6 \mathrm{ml})$ was filtered through a prewetted $0.45 \mu \mathrm{m}$ pore-size BA85 nitrocellulose filter (Schleicher \& Schuell) for protein binding. Proteins were eluted by addition of $40 \mu \mathrm{l}$ of $2 \times$ SDS sample buffer ${ }^{27}$ and heating to $65^{\circ} \mathrm{C}$ for $30 \mathrm{~min}$. For quantification of intracellular FlgM, the pelleted cells were resuspended in $50 \mu \mathrm{l}$ $1 \times$ SDS sample buffer and boiled for $10 \mathrm{~min}$. Pellet and supernatant fractions were adjusted to equal volumes (corresponding to $20 \mathrm{D}$ units for the cells), $10 \mu \mathrm{l}$ samples were loaded onto SDS-PAGE gels and FlgM was detected by immunoblotting, essentially as described ${ }^{23}$.

ATP measurement. ATP was measured using the firefly luciferase assay (timestable ATP determination kit, Biaffin) and the sample-processing procedures of ref. 24. Following resuspension in the media with or without CCCP, cells were incubated at $37^{\circ} \mathrm{C}$ for the times indicated, then 2-ml samples were withdrawn and mixed with $2 \mathrm{ml}$ of ice-cold $6 \% \mathrm{HClO}_{4}, 2.5 \mathrm{mM} \mathrm{Na} \mathrm{HPO}_{4}, 1 \mathrm{mM} \mathrm{KCl}$. Samples were left on ice for $30 \mathrm{~min}$ then centrifuged in the cold to remove denatured protein. Supernatants were brought to $\mathrm{pH} 7.0$ with $2 \mathrm{M} \mathrm{KOH}$, $0.3 \mathrm{M}$ MOPS, then frozen at $-20^{\circ} \mathrm{C}$, quickly thawed, and immediately centrifuged at $4{ }^{\circ} \mathrm{C}$ to remove crystals of $\mathrm{KClO}_{4}$. Samples were kept on ice and used for ATP determination within $2 \mathrm{~h}$. For ATP measurements, $50 \mu \mathrm{l}$ of supernatant was mixed with an equal volume of luciferase reagents in a 96-well plate and the luminescence was measured at $560 \mathrm{~nm}$ using a TopCount NXT Microplate scintillation and luminescence counter (Packard).

FlgE/ $\boldsymbol{\beta}$-lactamase activity. FlgE/ $\beta$-lactamase assays were performed essentially as described by in ref. 13, with minor modifications. Briefly, 1-ml cultures in LB were grown overnight at $37^{\circ} \mathrm{C}$. Cultures were diluted 100 -fold into $3 \mathrm{ml} \mathrm{LB}$ and grown at $37^{\circ} \mathrm{C}$ to a $D_{600}=0.5-0.7$, then diluted 50 -fold into fresh LB media containing ampicillin in a series of dilutions ranging from $800 \mu \mathrm{g} \mathrm{ml}^{-1}$ to $1.56 \mu \mathrm{g} \mathrm{ml}^{-1}$. The $D_{600}$ of each sample was measured after $4.5 \mathrm{~h}$ of further growth at $37^{\circ} \mathrm{C}$, and the minimum inhibitory concentration (MIC) was taken as the lowest ampicillin concentration giving $D_{600}<0.05$. For the experiment that measured the effect of CCCP, the flgE-bla gene was expressed from the ara promoter, induced with arabinose $30 \mathrm{~min}$ before addition of CCCP, to minimize FlgE/Bla accumulation in the periplasm before the addition of the uncoupler. Staining. Flagellar immunostaining was performed as described in ref. 26.

Measurement of FlgE-Bla in periplasm. Spheroplast preparation, periplasmic fractionation and immunoblot detection of FlgE-Bla in the cellular and periplasmic fractions was performed as described in ref. 25. 